\title{
Report of a rare and aggressive case of oral malignant melanoma
}

\author{
Fatemeh Ahmadi-Motamayel • Parisa Falsafi • \\ Fahimeh Baghaei
}

Received: 6 August 2011 / Accepted: 3 January 2012 / Published online: 25 February 2012

(C) Springer-Verlag 2012

\begin{abstract}
Background Oral melanoma is a very rare malignancy with unknown etiology. Its higher incidence is between 41 and 60 years of age. A high localization of oral melanoma was found in the maxilla. Gender distribution was reported as $1: 1$. Oral melanoma represents $0.2 \%$ to $8 \%$ of the all the total cases of melanoma of the body.

Case report In this article we report an aggressive case of oral melanoma in a 71-year-old male with chief complaint of black swelling of the gingiva, paresthesia, and denture ill fitting. Discussion Most oral melanomas are asymptomatic and painless in early stages and unfortunately diagnosis delayed until symptoms occurred. Early detection of suspected melanotic lesions by patient, dentist, and physician is very important.
\end{abstract}

\footnotetext{
F. Ahmadi-Motamayel

Research Center for Molecular Medicine, Department of Oral

Medicine, Hamadan University of Medical Sciences,

Hamadan, Iran

P. Falsafi

Department of Oral Medicine, Hamadan University of Medical

Sciences,

Hamadan, Iran

F. Baghaei

Department of Oral Pathology, Hamadan University of Medical

Sciences,

Hamadan, Iran

F. Ahmadi-Motamayel ( $\square)$

Research Center for Molecular Medicine, Department of Oral

Medicine, Hamadan Dental School Hamadan University of

Medical Science,

Hamadan, Iran

e-mail: Ahmadimotemayel@umsha.ac.ir
}

Keywords Oral $\cdot$ Melanoma $\cdot$ Malignant

\section{Background}

Oral melanoma is a very rare, infrequent, highly malignant, and aggressive neoplasm [1-5] with unknown etiology [1, $3,6,7]$ and incidence of 1.2 cases per ten million per year [2]. Although in the past 30-40 years, incidence has risen markedly and continues to increase in the USA, Canada, Australia, Asia, and Europe [5], the main prevalence of melanomas was among whites and [2] mucosal melanoma also is more common in the Japanese population (22-32\% of all melanomas [3]).

The first case of oral melanoma was reported at 1885 in the English literature [5]. A high localization was found in the maxillary gingival, hard palate, and alveolar ridge, with $80 \%$ of the cases reported in those sites and in about $20 \%$ of the cases it was localized in the mandible $[1-4,8,9]$. Oral melanoma occurs between 30 and 90 years of age with high occurrence in the sixth decade of life, as well as in some studies, it has been shown that its higher incidence is between 41 and 60 years of age [1-3].

In some literatures gender distribution was reported as 1:1 [5] and in other literatures male to female ratio was reported 2:1 [1, 10]. In general it does not have gender preference although some authors refer that incidence is slightly higher in men [1]. Oral melanoma represents $50 \%$ of all mucosal melanomas [5], and more than $20 \%$ of all melanomas of the head and neck region [9], $0.2 \%$ to $8 \%$ of the all the total cases of melanoma of the body [4], 1.6\% of all head and neck malignancies [11], 1.3\% of all cancers [8], and $0.5 \%$ of all oral malignancies $[1,4]$ in the world literature. 
The oral melanomas symptoms may include swelling, ulceration, pigmentation, discomfort, painful sensation, bleeding, ill-fitting dentures, and any combination of these symptoms [5]. In oral examination the oral melanoma may be presented as lesion with asymmetry, irregular border, uniform or mix color, light to dark brown, black, blue, and red when is non melanotic, with size more than $6 \mathrm{~mm}$, smooth or elevated lesion $[1,11]$.

The oral melanoma has low survival, and its prognosis is poor and worse than that of cutaneous melanoma $[1,11]$. Usually metastasis was found in many cases $[2,3,12,13]$. The main prognostic factor appears to be lymph node compromise [3]. The 5-year survival rate ranges from $5 \%$ to $20 \%[4]$.

\section{Case report}

A 71-year-old male farmer referred to the department of oral medicine with chief complaint of black swelling of gingiva and occasional paresthesia and denture fitness in the left upper gums. The swelling was present for the last 2 months, and the lesion size was increased in this period. He was referred two times for treatment to general physician and dentist, but only chlorhexidine mouth wash has been prescribed. He did not have any pigmented skin lesion and no systemic disease, sign, and symptom. He has used dentures about 30 years ago, and his denture is ill fitted now. He had paresthesia of the upper left lip about 2 weeks ago. In extraoral examination the patient did not have lymphadenopathy and the regional lymph nodes were not palpable. The patient did not have other problems.

In intraoral examination we observed a tumor-like exophitic, broad base sessile, smooth surface, gray to black color, rubbery consistency, $2 \times 2 \times 2-\mathrm{cm}$ dimension lesion located on the left anterior buccal aspect of the edentulous ridge of the maxilla, and another same amelanotic pink lesion located on the posterior side of this lesion with ulcerated surface. A popular, sessile, verrucous surface, firm consistency, $1 \times 1 \times 1-\mathrm{cm}$ dimension lesion also was located near of the perio lesion on the left maxillary buccal mucosa. Multiple asymmetric melanotic macules with irregular borders, brown to black color, 0.5 to $2 \mathrm{~cm}$ in diameter were located on all of the hard and soft palate. In palpation the patient did not reveal any tenderness and bleeding (Figs. 1 and 2).

In panoramic and periapical radiography, involvement of the underlying bone was detected. Bone resorption was radiolucent with nonsclerotic and well-defined borders and $2 \times 3 \mathrm{~cm}$ observed under the tumor-like lesion (Figs. 3 and 4).

Result of the incisional biopsy showed that there was malignant cellular proliferation of melanocytes in all areas of connective tissue. Junctional activity also was observed on the junctional area. Nuclear shape varies from spindle to

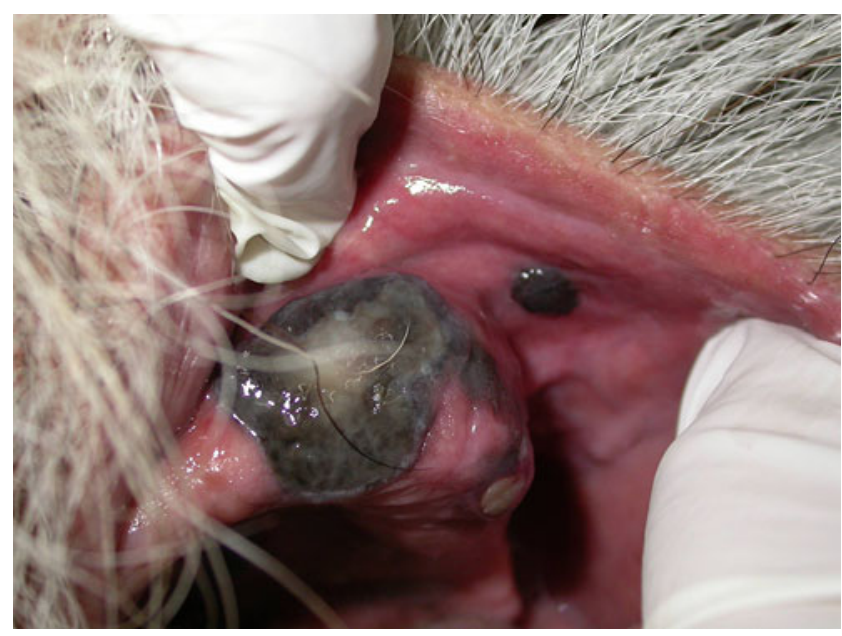

Fig. 1 A tumor-like exophitic, sessile, smooth surface, gray to black color and amelanotic pink lesion on the left anterior buccal aspect of edentulous ridge of maxilla. Another popular, sessile, verrucous surface lesion on the left maxillary buccal mucosa

oval and circular shape. Melanin pigment also was observed in some cells and confirmed the diagnosis of malignant melanoma (Figs. 5 and 6).

The patient was referred for the whole body scan and oral and maxillofacial department for treatment, but he and his family did not accept to follow the treatment. After 3 months he was referred to the oncology department because he cannot breathe and eat anything. His oral lesion grew rapidly to about $10 \times 10 \times 10 \mathrm{~cm}$, chemotherapy and radiotherapy regimen were used just for palliative therapy.

\section{Discussion}

This patient does not have any symptom and pain until his denture was ill fitted and he has seen the black change and

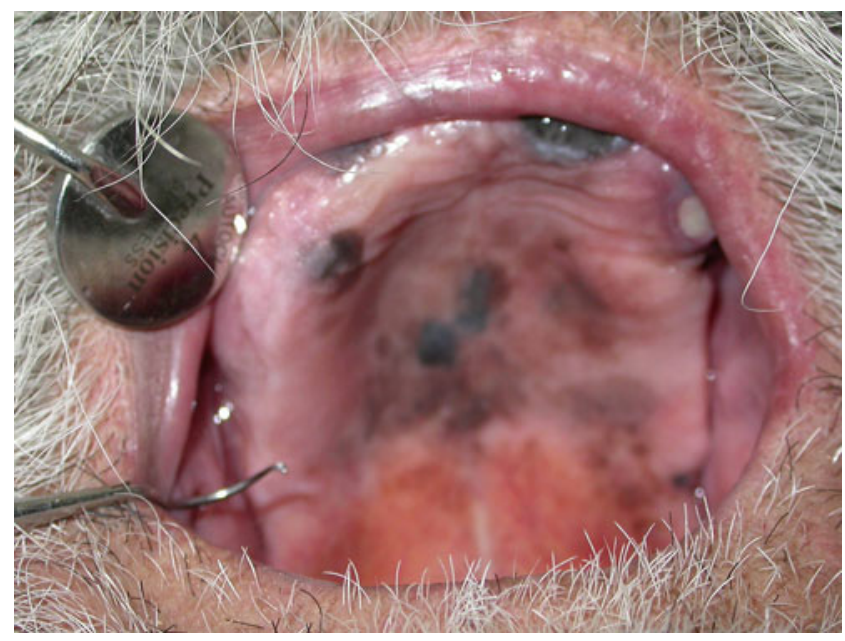

Fig. 2 Multiple asymmetric melanotic macules with irregular borders, brown to black color, 0.5 to $2 \mathrm{~cm}$ in diameter on all of the hard and soft palate 


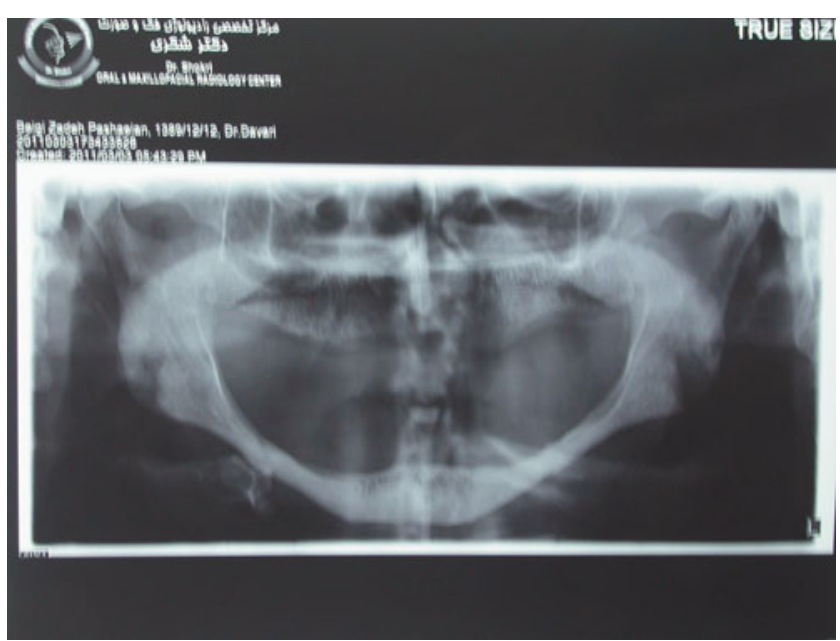

Fig. 3 Involvement of the underlying bone in panoramic radiography

rapidly growing mass in the gingiva. Unfortunately since oral melanoma is painless usually [1], patients delay referring to the dentist until frequent symptoms such as denture problem, ulceration, bleeding, growth, dental mobility pain, and paresthesia occur [1]. Thus most of oral melanomas are in an advanced stage at the time of diagnosis because it is asymptomatic in the early stages $[5,9]$.

He started using dentures about 30 years ago and had some problem with denture use, but he did not go to a doctor to have his dentures changed and to solve his problems. One study reported relation between prosthetic microtrauma and oral melanoma in $60 \%$ of the cases although there are no any obvious data on the effect of denture trauma on oral melanoma [1].

Early detection and appropriate treatment are important factors in prognosis $[1,14]$. Immediate diagnosis of melanotic changes in oral mucosa is very important. This patient referred to a physician and dentist, and only a mouth wash

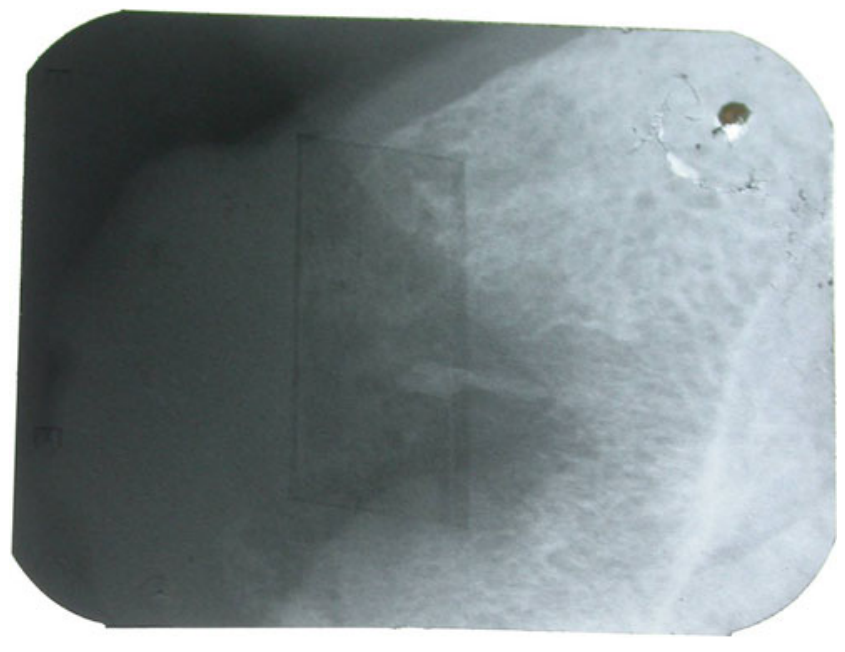

Fig. 4 In periapical radiography radiolucent bone resorption with nonsclerotic and well-defined borders and $2 \times 3 \mathrm{~cm}$ under the tumorlike lesion

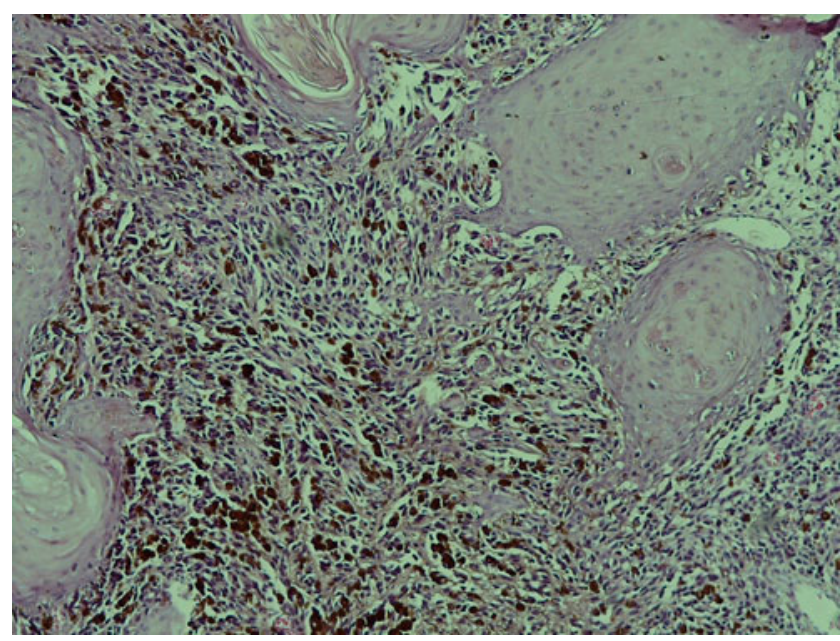

Fig. 5 There is junctional activity from epithelium into connective tissue and the epithelium shows pseudoepitheliomatous hyperplasia in histopathologic view

has been prescribed, thus more education in the field of oral high risk lesions is very necessary to general physicians and dental practitioners.

The GREENE criteria in 1953 for primary oral melanoma consist of: melanoma in the oral mucosa, junctional activity, and any extraoral primary melanoma [1]. Oral melanoma may be primary, but long-distance metastasis was reported in $60 \%$ of the cases [2], the most common site being the lungs [2]. Same to other studies, our patient's lesions were located in the palate and upper alveolar ridge without any other oral region involvement and metastasis [2].

Mucosal melanoma behavior, treatment and prognosis are different from cutaneous melanoma [5]. Prognosis of oral melanoma is extremely poor and is also most deadly in comparison to other organ neoplasms [8], as well as

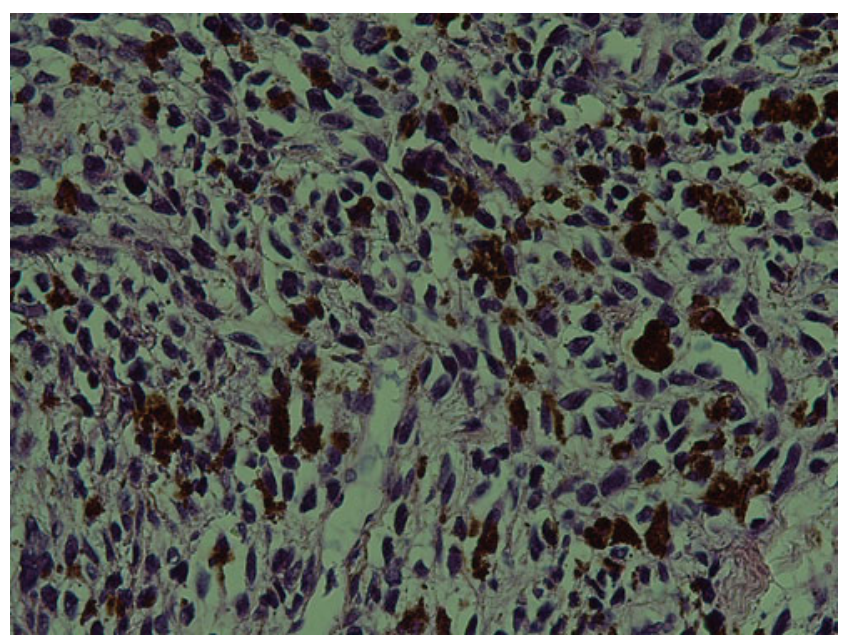

Fig. 6 Malignant spindled or ovoid melanocytes with hyperchromatic and pleomorphic nuclei and considerable amount of melanin are present in histopathologic view 
vascular or lymphatic invasion that facilitates spreading of melanoma to other sites is a more important prognostic factor [2]. The 5 -year survival rate is reported to be $0-50 \%[5$, 12]; in another study, 5-year survival from the head and neck melanoma was reported to be $5 \%$ [5]. Studies showed more prevalence of mucosal melanoma in male than in female; our patient's gender also was male [5].

Although some studies showed that the main treatment is surgical resection [11], treatment protocol of oral melanoma consists of surgical resection, neck dissection for lymph node metastasis, cryotherapy, chemotherapy, radiotherapy, and immunotherapy, or combination of these therapies $[2,4,7,12]$. Some studies suggest that biopsy should not be performed because it may cause metastasis [12]; in this patient because of the existence of amelanotic lesion and large bone resorption, we did an incisional biopsy of the suspected area. Other studies also suggest that large lesions should be sampled with incisional biopsies [5]; another study also reported no correlation between incisional biopsy and distant metastasis [7]. One study showed that one third of mucosal melanomas may be amelanotic. Our patient also had an amelanotic lesion.

Same to the more common site of oral melanoma, the primary lesion of this patient is located on the maxillary area. Although secondary or metastatic lesion may be located on the tongue, parotid, and tonsils [1], the nodular form of oral melanoma with a vertical growth is more aggressive than the other forms. This patient also had exophitic and nodular melanotic changes [1]. Incidence peak of oral melanoma is between the fifth and sixth decades of life [15], and mean age at diagnosis of patients with oral melanoma is 55 years [5]. This patient also was 71 years old. A higher incidence of oral melanoma is reported in India, Africa, and Japan than in western countries $[1,2,12]$. Race, culture, and geographic factors may influence oral melanoma [5]. Although this patient was a farmer, there are no confirmed data that UV radiation influences development of oral melanoma [1].

In 1995 it was noted that oral melanoma is separate from cutaneous melanoma $[1,7,16]$ as well as oral melanoma may not have the $\mathrm{ABCDE}$ characteristics of skin melanoma, but in our patient all of the $\mathrm{ABCDE}$ criteria were present. Differential diagnosis consists of Addison's disease, Peutz-Jeghers' syndrome, Kaposi's sarcoma, melanotic pigmentations, and melanoacanthoma [1], but our patient did not have any other signs and symptoms suggesting these lesions.

According to the department of maxillofacial pathology, the proposed classification upon histological pattern oral melanoma is divided as melanoma in situ, invasive, and combined [7]; our patient's lesion was an invasive form. However because of rarity of oral melanoma, data on epidemiology, tumor behavior, treatment, follow-up, and survival of patients are mainly based on single case reports $[7,17]$.

\section{Conclusion}

Most oral melanomas are asymptomatic and painless in early stages, and unfortunately, diagnosis is delayed until symptoms such as swelling, ulceration and bleeding occur. Because early diagnosis is very important for better survival and prognosis in oral melanoma, thus early detection of oral melanoma is very critical, whose goal will be achieved by self-examination training and early detection of suspected melanotic lesions by dentist and physicians.

Conflicts of interest The authors declare that they have no conflicts of interest.

\section{References}

1. Aguas SC, Quarracino MC, Lence AN, Lanfranchi-Tizeira HE (2009) Primary melanoma of the oral cavity: ten cases and review of 177 cases from literature. Med Oral Patol Oral Cir Bucal 14(6): E265-E271

2. Lourenco SV, Sangueza MA, Sotto MN, Bologna SB, Giacomo TB, Buim ME et al (2009) Primary oral mucosal melanoma: a series of 35 new cases from South America. Am J Dermatopathol 31(4):323-330

3. Lourenco SV, Bologna SB, Colucci F, Neto CF, Montenegro FL, Nico MM (2010) Oral mucosal melanoma of the mandibular gingiva: a case report. Cutis 86(2):89-93

4. Tucci R, Aburad De Carvalhosa A, Anunciacao G, Daumas Nunes F, Dos Santos Pinto D Jr (2010) Late diagnosis of a primary oral malignant melanoma: a case report. Minerva Stomatol 59(1-2):55-59

5. Hashemi Pour MS, Rad M, Zarei MR, Chamani G (2009) Malignant mucosal melanoma of the head and neck diagnosed in an Iranian population over an 11-year period. Am J Appl Sci 6 (8): 1467

6. Lessa NL, Moleri AB, Merly F, Moreira LC, Moreira MJ, Antunes HS (2008) Oral melanoma: an unusual presentation. Dermatol Online J 14(1):17

7. Meleti M, Leemans CR, Mooi WJ, van der Waal I (2007) Oral malignant melanoma: the Amsterdam experience. J Oral Maxillofac Surg 65(11):2181-2186

8. Gondivkar SM, Indurkar A, Degwekar S, Bhowate R (2009) Primary oral malignant melanoma - a case report and review of the literature. Quintessence Int 40(1):41-46

9. Hashemi Pour MS (2008) Malignant melanoma of the oral cavity: a review of literature. Indian J Dent Res 19(1):47-51

10. Luna-Ortiz K, Campos-Ramos E, Pasche P, Mosqueda-Taylor A (2011) Oral mucosal melanoma: conservative treatment including laser surgery. Med Oral Patol Oral Cir Bucal 16(3):e381-e385

11. Bujas T, Pavic I, Prus A, Marusic Z, Balicevic D (2010) Primary oral malignant melanoma: case report. Acta Clin Croat 49(1):55-59 
12. Umeda M, Murata M, Suzuki H, Yanagida T, Shibuya Y, Komori T (2010) A case of malignant melanoma of the oral cavity alive with liver metastasis for a long period with administration of a biologic response modifier, OK432. Kobe J Med Sci 56(3):E140-E147

13. Gauwerky KJ, Ehrenfeld M, Bakos RM, Volkenandt M, Ruzicka T, Berking C (2010) A rare case of local relapsing oral melanoma. J Dtsch Dermatol Ges 8(8):614-616

14. Devi P, Bhovi T, Jayaram RR, Walia C, Singh S (2011) Malignant melanoma of the oral cavity showing satellitism. J Oral Sci 53 (2):239-244
15. Jahanbani J, Forouzandeh A, Sadri D, Mirlashari J (2008) Oral malignant melanoma diagnosed in an Iranian population over an 11-year period. Oral Maxillofac Surg 12(4):181-183

16. Moreira RN, Santos CR, Lima NL, Verli FD, Marinho SA (2010) Oral and cutaneous melanoma: similarities and differences. J Clin Med Res 2(4):155-158

17. Hebishima T, Matsumoto Y, Watanabe G, Soma G, Kohchi C, Taya K et al (2011) Oral administration of immunopotentiator from Pantoea agglomerans 1 (IP-PA1) improves the survival of B16 melanoma-inoculated model mice. Exp Anim 60 (2):101-109 